Pacific Journal of Mathematics

HÖLDER CONTINUITY OF THE GRADIENT AT A CORNER
FOR THE CAPILLARY PROBLEM AND RELATED RESULTS CRY M. LIEBERMAN 


\title{
HÖLDER CONTINUITY OF THE GRADIENT AT A CORNER FOR THE CAPILLARY PROBLEM AND RELATED RESULTS
}

\section{GARY M. LIEBERMAN}

\begin{abstract}
It is well-known that solutions of the capillary problem are smooth when the boundary and contact angle are smooth. Using fairly deep methods which are specific to the capillary problem, Simon and Tam have proved the smoothness of the solution at a corner. Here the smoothness is considered in the context of general nonlinear boundary value problems. The primary tool is a maximum principle argument.
\end{abstract}

Let $\Omega$ be a bounded domain in $\mathbf{R}^{2}$ with unit inner normal $\gamma$, let $\kappa$ and $\phi$ be positive constants with $\phi<\pi$, and consider the problem

$$
\begin{gathered}
\operatorname{div}\left(\left(1+|D u|^{2}\right)^{-1 / 2} D u\right)=\kappa u \quad \text { in } \Omega, \\
\left(1+|D u|^{2}\right)^{-1 / 2} D u \cdot \gamma=\cos \phi \quad \text { on } \partial \Omega .
\end{gathered}
$$

When $\partial \Omega$ is sufficiently smooth, it is well-known that $(0.1)$ has a unique, smooth solution. Specifically, $\partial \Omega \in C^{2, \alpha}$ implies $u \in C^{2, \varepsilon}(\bar{\Omega})$ for some $\varepsilon>0$ by [7], [27] (in fact $\varepsilon=\alpha$ by [15, Lemma $2^{\prime}$ ]) while $\partial \Omega \in C^{1,1}$ implies $u \in C^{1, \beta}$ for any $\beta<1$ by [7], [20], [27]. If $\phi$ is suitably restricted, $(0.1)$ has a unique solution (in an appropriate weak sense) even for nonsmooth domains (see [5], [6]). Under various hypotheses, this solution may be unbounded [3] or bounded but discontinuous [12]. Our interest here is with circumstances under which $u$ will be $C^{1}$ : We assume that $\partial \Omega$ is the union of finitely many smooth curves which meet at an angle $\theta$ in the range $(0, \pi)$. If $\theta>|2 \phi-\pi|$ (which is easily seen to be necessary for $(0.1)$ to have a $C^{1}$ solution), Simon [25] has shown that $u \in C^{1}$. We improve this result by showing that $u \in C^{1, \varepsilon}$ for some computable $\varepsilon$ and by considering more general differential equations and boundary conditions. Moreover our method rests on a simple application of the maximum principle, and we can prove regularity results in more than two dimensions. A related argument was used by Miersemann [23] (in two dimensions) when the quantity $\kappa u$ in $(0.1)$ is replaced by a constant to prove $C^{1, \varepsilon}$ regularity in a corner. His method does not readily extend to $(0.1)$ but ours includes his situation. The biggest differences 
between the two approaches are that we use essentially only the elliptic structure of $(0.1)$ to produce an auxiliary mixed boundary value problem while Miersemann uses the divergence structure of $(0.1)$ to produce a Dirichlet problem.

We begin in $\S 1$ with a related $C^{1}$ regularity result for nonlinear boundary value problems in Lipschitz domains and an application to free boundary problems. Although the results of this section do not bear directly on the capillary problem, the method of proof is relevant because it demonstrates a key point: introduction of a nonlinear combination of derivatives of the solution via a variant of Giusti's barrier construction for the Dirichlet problem [10]. Next we introduce an auxiliary boundary value problem in $\S 2$ in order to study a general nonlinear boundary value problem for uniformly elliptic equations modelled on the capillary problem in a corner with a gradient bound. In $\S 3$, we consider the two-dimensional capillary problem in a corner for which gradient estimates were proved by Korevaar [13]. We show that his conditions for a gradient bound imply also Hölder continuity of the gradient, and a strong result for weak solutions of certain nonlinear boundary problems in divergence is proved using the perturbation argument of Giaquinta and Giusti [8]. In the final section, we prove Hölder continuity of the gradient near an edge for uniformly elliptic problems. Because Korevaar's gradient bound is also valid in this case, we infer regularity for the higher-dimensional capillary problem in a domain with edges.

An interesting comparison can be made between the proof of the continuity of the gradient at a corner given here and the proof of the boundedness of the gradient given by Korevaar. Here we work directly in the corner while Korevaar approximates the corner. It might be useful to know if Korevaar's method can be applied directly and if our method gives uniform continuity of the gradient in approximating domains.

1. Nonlinear boundary value problems in Lipschitz domains. Let $\Omega$ be a bounded domain in $\mathbf{R}^{2}$ and consider the boundary value problem

$$
\text { (1.1) } \quad F\left(x, u, D u, D^{2} u\right)=0 \quad \text { in } \Omega, \quad G(x, u, D u)=0 \quad \text { on } \partial \Omega \text {. }
$$

We are interested in the behavior of solutions of (1.1) near the boundary under various hypotheses on $F, G$, and $\Omega$. For example, in this section we assume $G$ to be continuous while later sections consider discontinuous $G$. 
To state our hypotheses, we introduce some notation. We write $\mathbf{S}^{2}$ for the set of all $2 \times 2$ real, symmetric matrices and $\Gamma=\Omega \times \mathbf{R} \times \mathbf{R}^{2} \times \mathbf{S}^{2}$. A typical element of $\Gamma$ is written as $X=(x, z, p, r)$. Subscripts and superscripts are used to indicate partial derivatives, components of vectors and tensors, and enumeration of quantities; the usage should be clear from the context. For example

$$
\begin{gathered}
x^{i}=i \text { th component of } x, \quad p_{j}=j \text { th component of } p, \\
F_{r}=\frac{\partial F}{\partial r}, \quad F_{z}=\frac{\partial F}{\partial z}, \\
F_{i}=\frac{\partial F}{\partial x^{i}}, \quad F^{i}=\frac{\partial F}{\partial p_{i}}, \quad F^{i j}=\frac{\partial F}{\partial r_{i j}} .
\end{gathered}
$$

Also we use $d$ to denote the distance function:

$$
d(x)=\inf _{y \in \partial \Omega}|x-y|,
$$

and, for $x_{0} \in \partial \Omega$ and $R>0$, we define

$$
\Omega_{R}=\left\{x \in \Omega:\left|x-x_{0}\right|<R\right\}, \quad \Sigma_{R}=\left\{x \in \partial \Omega:\left|x-x_{0}\right|<R\right\} .
$$

Our basis hypotheses are that there are positive constants $\alpha<1$, $\mu \geq 1, \mu_{0}, \mu_{1}, \mu_{2}$ such that

$$
\begin{gathered}
|\xi|^{2} \leq F^{i j} \xi_{i} \xi_{j} \leq \mu|\xi|^{2} \\
|F(x, z, p, 0)| \leq \mu_{0} d^{\alpha-1} \\
\left|F_{x}\right|+|p|\left|F_{z}\right|+|r|\left|F_{p}\right| \leq \mu_{1} d^{\alpha-2}+\mu_{2}|r|^{2}
\end{gathered}
$$

for all $X \in \Gamma$ and $\xi \in \mathbf{R}^{2}$. If we also suppose that there are positive constants $\nu$ and $\nu_{1}$ such that

$$
\text { (1.3a) }\left|G(x, z, p)-G\left(x^{\prime}, z^{\prime}, p^{\prime}\right)\right| \leq \nu\left(\left|x-x^{\prime}\right|+\left|z-z^{\prime}\right|\right)^{\alpha}+\nu_{1}\left|p-p^{\prime}\right|
$$

and a unit vector $\sigma(x, z)$ such that

$$
\liminf _{t \rightarrow 0^{+}} \frac{G(x, z, p+t \sigma)-G(x, z, p)}{t} \geq 1
$$

for all $(x, z, p)$ and $\left(x^{\prime}, z^{\prime}, p^{\prime}\right)$ in $\partial \Omega \times \mathbf{R} \times \mathbf{R}^{2}$, we can prove stronger versions of the results in [19].

THEOREM 1.1. Let $\partial \Omega \in C^{0,1}$, let $u \in C^{0,1}(\bar{\Omega}) \cap W_{\text {loc }}^{3,2}(\Omega)$ and suppose $|u|+|D u| \leq K$. Suppose also that $u$ is a solution of (1.1) for functions $F$ and $G$ satisfying (1.2), (1.3) as long as $|z|+|p|,\left|z^{\prime}\right|+\left|p^{\prime}\right| \leq K+1$. 
Then there are positive constants $\gamma=\gamma(\mu)$ and $\sigma, C$ depending also on $K, \Omega, \nu_{1}, \mu, \mu_{2}$ such that

$$
\begin{aligned}
& |u|_{1, \sigma ; \Omega_{R / 2}}+\sup _{\Omega^{\prime} \subset \subset \Omega_{R / 2}} \operatorname{dist}\left(\Omega^{\prime}, \Sigma_{R}\right)^{1-\gamma+\sigma}|u|_{2, \gamma ; \Omega^{\prime}} \\
& \leq C\left(\mu_{0}+\mu_{1}+\nu+R^{-\sigma} \underset{\Omega_{R}}{\operatorname{osc}} D u\right) .
\end{aligned}
$$

Proof. As in [19], we introduce a mollification of the function $g=$ $G\left(x_{0}, u\left(x_{0}\right), \cdot\right)$. Because of an invalid assertion in that work (namely, that condition (1.3b) could be replaced by the inequality $\left|G_{p}\right| \geq 1$ ), we state this mollification explicitly. Let $\phi$ be a nonnegative $C^{2}\left(\mathbf{R}^{2}\right)$ function with $\phi\left(\left\{\left|p^{\prime}\right|>1\right\}\right)=\{0\}$ and $\int_{\mathbf{R}^{2}} \phi=1$, and set

$$
h(p, t)=\int_{\mathbf{R}^{2}} g\left(p+t p^{\prime}\right) \phi\left(p^{\prime}\right) d p^{\prime}
$$

for $|p| \leq K,|t| \leq 1$. Then

$$
\begin{gathered}
h(p, 0)=g(p), \quad\left|h_{p}(p, t)\right|,\left|h_{t}(p, t)\right| \leq \nu_{1}, \\
\left|D^{2} h(p, t)\right| \leq C \nu_{1} / t, \quad\left|h_{p}(p, t)\right| \geq 1 .
\end{gathered}
$$

We also set $M=\sup _{\Omega_{R}}|g(D u)|$ and note that

$$
M \leq \nu_{1} \underset{\Omega_{R}}{\operatorname{osc}} D u .
$$

Next we infer from [22] that there are positive constants $\delta$ and $C$ and a function $w \in C^{0}(\bar{\Omega}) \cap C^{2}(\Omega)$ such that

$$
F^{i j} D_{i j} w \leq-d^{\delta-2}, \quad 0 \leq w \leq C d^{\delta} \text { in } \Omega .
$$

Then for $L_{0}$ a nonnegative constant and $f$ a nonnegative $C^{0}[0, \infty] \cap$ $C^{2}(0, \infty)$ function to be further specified, we set

$$
\begin{aligned}
v(x) & =h(D u, f(w))+\nu|x|^{\alpha}+M|x|^{2} R^{-2}+L_{0} f(w), \\
\Omega^{*} & =\Omega_{R} \cap\{f<1\}, \quad \mu^{\prime}=\mu_{0}+\mu_{1}+\nu .
\end{aligned}
$$

A simple calculation shows that $v \geq 0$ on $\partial \Omega^{*}$ if $L_{0} \geq(1+K) \nu_{1}$, and also there are constants $c_{0}$ and $c_{1}$ such that

$$
\begin{aligned}
F^{i j} D_{i j} v \leq & c_{0} \frac{|D v|^{2}}{f}+c_{1}\left(\mu^{\prime}+d^{\alpha-2}+M R^{-2}\right)-\left(L_{0}+h_{t}\right) f^{\prime} d^{\delta-2} \\
& +F^{i j} D_{i} w D_{j} w\left[\left(L_{0}+h_{t}\right) f^{\prime \prime}+c_{1}\left(1+\left(L_{0}+h_{t}\right)^{2}\right) \frac{\left(f^{\prime}\right)^{2}}{f}\right]
\end{aligned}
$$

With $f_{0}$ and $\varepsilon$ positive constants to be chosen, we take

$$
f=f_{0} w^{\varepsilon}, \quad L_{0}=1+\nu_{1}+\nu_{1} K,
$$


and we set

$$
Q v=F^{i j} D_{i j} v-c_{0} \frac{|D v|^{2}}{f}
$$

It follows that

$Q v \leq d^{\delta \varepsilon-2}\left(c_{1}\left(\mu^{\prime}+M R^{-\delta \varepsilon}\right)-\varepsilon f_{0}\right)+\varepsilon f_{0} w^{\varepsilon-2} F^{i j} D_{i} w D_{j} w\left(\left(c_{2}+1\right) \varepsilon-1\right)$.

Hence for

$$
\varepsilon=\frac{1}{1+c_{2}}, \quad f_{0}=2\left(1+c_{1}\right)\left(1+c_{2}\right)\left(\mu^{\prime}+M R^{-\delta \varepsilon}\right),
$$

we have $Q v<0$ in $\Omega^{*}$. Then the maximum principle implies that $v \geq 0$ in $\Omega^{*}$. The proof of [19, Theorem 1] allows us to infer (1.4) with $\sigma=\delta \varepsilon$.

A slight modification of Theorem 1.1 is necessary when

$$
G(x, z, p)=|p|-g(x, z) .
$$

Corollary 1.2. Suppose in Theorem 1.1 that conditions (1.3) are replaced by (1.5),

$$
\begin{gathered}
\left|g(x, z)-g\left(x^{\prime}, z^{\prime}\right)\right| \leq \nu\left(\left|x-x^{\prime}\right|^{\alpha}+\left|z-z^{\prime}\right|^{\alpha}\right), \\
|D u| \geq p_{0} \quad \text { in } \Omega_{R}
\end{gathered}
$$

for some positive constant $p_{0}$. Then (1.4) holds with $C$ depending also on $p_{0}$.

Proof. Now take $h(p, t)=\left(|p|^{2}+p_{0}^{2} t^{2}\right)^{1 / 2}-g\left(x_{0}, u\left(x_{0}\right)\right)$. Then $h_{p}=\left(|p|^{2}+p_{0}^{2} t^{2}\right)^{-1 / 2} p$, and hence $\left|h_{p}\right| \geq 1 / 2$. The proof is completed in the same way as for Theorem 1.1.

Corollary 1.2 provides an alternative proof of the regularity of the free boundary in the following situation. Let $\Gamma$ be a smooth Jordan curve and look for a domain $\Omega$, a curve $\Gamma^{*}$, and a function $u$ such that

$$
\begin{array}{ll}
\partial \Omega=\Gamma \cup \Gamma^{*}, & \Gamma \cap \Gamma^{*}=\varnothing, \\
\Delta u=0 \text { in } \Omega, & u=1 \text { on } \Gamma, \quad u=0 \text { and }|D u|=1 \text { on } \Gamma^{*} .
\end{array}
$$

(Obviously more general problems can be considered.) It is wellknown that $\Gamma^{*}$ is Lipschitz and the boundary condition implies that $|D u|$ is bounded away from zero near $\Gamma^{*}$. From Corollary 1.2 we see that $u$ is $C^{1, \sigma}$ near $\Gamma^{*}$ and hence that $\Gamma^{*} \in C^{1, \sigma}$. The theory of free boundary problems shows that $\Gamma^{*}$ is actually analytic; see [11, Chapter V]. We content ourselves with results involving smoothness of $D u$ and $D^{2} u$ only. 
Corollary 1.3. Suppose, in addition to the hypotheses of Theorem 1.1 , that $G_{p}$ is continuous with

$$
\left|G_{p}(x, z, p)-G_{p}\left(x, z, p^{\prime}\right)\right| \leq \nu_{1}\left|p-p^{\prime}\right|^{\eta}
$$

for some $\eta>0$. Then (1.4) is valid with $\sigma=\min \{\alpha, \delta(\Omega, \mu)\}$. Moreover if $\partial \Omega$ is $C^{1}$ or if $\Omega$ is convex, then $\sigma=\alpha$.

Proof. The bootstrap argument of [19, Theorem 2] shows that we can take $\varepsilon=\min \{1, \delta / \alpha\}$ in Theorem 1.1. When $\partial \Omega \in C^{1}$ or $\Omega$ is convex, [24] shows that we can take $\delta \in(\alpha, 1)$.

COROLlARY 1.4. In addition to the hypotheses of Theorem 1.1, suppose that

$$
\begin{gathered}
|F(x, z, p, 0)| \leq \mu_{0}, \\
\left|F_{x}\right|+\left|F_{z}\right|+|r|\left|F_{p}\right| \leq \mu_{1} d^{\alpha-1}+\mu_{2}|r|^{2},
\end{gathered}
$$

$\partial \Omega \in C^{1, \alpha}$, and $G \in C^{1, \alpha}\left(\partial \Omega \times R \times \mathbf{R}^{2}\right)$ with

$$
|D G(X)| \leq \nu_{1}, \quad\left|D G(X)-D G\left(X^{\prime}\right)\right| \leq \nu_{2}\left|X-X^{\prime}\right|^{\alpha} .
$$

Then there are positive constants $\gamma=\gamma(\alpha, \mu)$ and $C$, depending also on $K, \Omega, \mu, \mu_{0}, \mu_{1}, \mu_{2}, \nu_{1}$ and $\nu_{2}$, such that $u \in C^{2, \gamma}\left(\Omega_{R / 2}\right)$ and

$$
\left|D^{2} u\right|_{0 ; \Omega_{R / 2}}+R^{\gamma}\left[D^{2} u\right]_{\gamma ; \Omega_{R / 2}} \leq C\left(1+\underset{\Omega_{R}}{\operatorname{osc}} D u / R\right) .
$$

Proof. Let $\rho$ be the regularized distance of [16], so that

$$
\rho>0, \quad \rho / d<C, \quad d / \rho<C, \quad\left|D^{2} \rho\right| \leq C d^{\alpha-1} \quad \text { in } \Omega
$$

and $|\rho|_{1, \alpha} \leq C$. Now extend $G$ to a $C^{1, \alpha}\left(R \times \mathbf{R} \times \mathbf{R}^{2}\right)$ function satisfying (1.10) (with possibly different, but controlled, constants $\nu_{1}, \nu_{2}$ ), let $\bar{G}(X, t)$ be the mollification of $G$ from [19, Lemma 3], and set

$$
M=\sup _{\Omega_{R}}|G(x, u, D u)|, \quad g(x)=\bar{G}(x, u, D u, \rho) /(1+M / R) .
$$

From Corollary 1.3 and (1.12) we infer that

$$
g=0 \quad \text { on } \Sigma_{R}, \quad\left|F^{i j} D_{i j} g\right| \leq c d^{\beta-1} \text { in } \Omega_{R}
$$

for any $\beta \in(0, \alpha)$. It then follows from Dirichlet problem results [18, Sect. 5] that $g \in C^{1, \gamma}\left(\Omega_{3 R / 4}\right)$ with

$$
|D g|_{0 ; \Omega_{3 R / 4}}+R^{-\gamma}|D g|_{\gamma ; \Omega_{3 R / 4}} \leq C .
$$

As in [19, Theorem 3], this estimate leads to (1.11). 
2. Nonlinear boundary value problems in corners. We now study (1.1) when $\partial \Omega$ is smooth except at isolated points and $G$ is suitably discontinuous at those points. Specifically we let $\alpha<1$ and $\theta_{0}<\pi$ be positive constants and suppose that, in the terminology of the previous section, $\Sigma_{1}$ consists of two $C^{1, \alpha}$ curves $\Sigma^{1}$ and $\Sigma^{2}$ which meet only at the origin in an angle $\theta_{0}$. Writing $\gamma_{i}$ for the inner unit normal to $\Omega$ on $\Sigma^{i}$ and $b^{i}$ for the restriction of $G$ to $\Gamma_{i}=\left(\Sigma^{i} \backslash\{0\}\right) \times \mathbf{R} \times \mathbf{R}^{2}$, we suppose that

$$
\begin{gathered}
b_{p}^{i} \cdot \gamma_{i} \geq 1 \text { on } \Gamma_{i}, \\
b^{i} \in C^{1, \alpha}\left(\Gamma_{i}\right),
\end{gathered}
$$

$$
\left|\operatorname{det}\left(\frac{\partial b^{i}}{\partial p_{j}}\left(X_{i}\right)\right)\right| \geq \varepsilon \text { for } X_{i} \in \Gamma_{i} \text { with }\left|X_{1}-X_{2}\right|<\varepsilon_{0} \text {, }
$$

$$
\left|b^{i}(X)-b^{i}\left(X^{\prime}\right)\right| \leq \nu_{1}\left|X-X^{\prime}\right| \text { for } X \text { and } X^{\prime} \text { in } \Gamma_{i} .
$$

Note that $(2.1 \mathrm{~d})$ follows from the smoothness of the $b$ 's and the functional independence of $b^{1}(0, z, \cdot)$ and $b^{2}(0, z, \cdot)$; however, this quantification will be useful later. In addition, an obvious necessary condition for solutions of (1.1) to be $C^{1}$ at 0 is that

(2.2) there is $p^{*} \in \mathbf{R}^{2}$ such that $b^{1}\left(0, u(0), p^{*}\right)=b^{2}\left(0, u(0), p^{*}\right)=0$.

The main result of this section is that (2.2) is sufficient. In the next section we relate this condition to the capillary problem, but we note here that (2.1) and (2.2) imply that $D u(0)$ must be $p^{*}$ if $u \in C^{1}$.

THEOREM 2.1. Let $\Omega$ be as described above, let $u \in C^{0,1}(\bar{\Omega}) \cap W_{\text {loc }}^{3,2}(\Omega)$ and suppose $|u|+|D u| \leq K$. Suppose also that $u$ is a solution of $(1.1)$ for functions $F$ and $G$ satisfying (1.2a), (1.9), (2.1), (2.2). Then Du(0) exists and there are constants $C, \sigma$ depending only on $\mu, \mu_{0}, \mu_{1}, \nu_{1}, \varepsilon$, $\varepsilon_{0}, \theta_{0}$ such that

$$
|D u(x)-D u(0)| \leq C\left(1+\underset{\Omega_{1}}{\operatorname{osc}} D u\right)|x|^{\sigma} .
$$

Proof. By considering $D u-p^{*} \cdot x$ in place of $u$, we may assume that $p^{*}=0$. Let $R \leq \varepsilon_{0} /(2+2 K)$ and, for $\phi$ as in Theorem 1.1, set

$$
h(p, t)=\int b^{1}\left(0, u(0), p-\frac{1}{2} \varepsilon_{0} t p^{\prime}\right) \phi\left(p^{\prime}\right) d p^{\prime} .
$$


With $f$ and $w$ functions to be further specified and $L_{0}$ a nonnegative constant, we set

$$
v(x)=h(D u, f(w))+L_{0} f(w) .
$$

Our analysis of $v$ in $\Omega_{R} \cap\{f<1\}$ and on $\Sigma_{1}$ and $\{f=1\}$ is essentially the same as that in Theorem 1.1, so we focus on the situation on $\Sigma^{2} \cap\{f<1\}$.

Let $\tau=\left(-\gamma_{2}^{2}, \gamma_{2}^{1}\right)$ be the tangent vector to $\Sigma^{2}$ and, for $T$ to be chosen, we introduce the operator

$$
M^{*}=T \tau^{i} D_{i}+\gamma_{2}^{i} D_{i}
$$

on $\Sigma^{2}$. According to Corollary 1.3, $u \in C^{2}\left(\Omega \cup \Sigma^{2} \backslash\{0\}\right)$ and therefore we can differentiate the equation $b^{2}=0$ along $\Sigma^{2}$ to obtain

$$
0=b_{p_{i}}^{2} \tau^{k} D_{i k} u+b_{z}^{2} \tau \cdot D u+b_{x}^{2} \cdot \tau \text {. }
$$

From the differential equation, we see that there is a symmetric matrix $\left(a^{i j}\right)$ such that

$$
\begin{gathered}
|\xi|^{2} \leq a^{i j} \xi_{i} \xi_{j} \leq \mu|\xi|^{2}, \\
a^{i j} D_{i j} u=-F(x, u, D u, 0) \text { on } \Sigma^{2}
\end{gathered}
$$

We now set

$$
\begin{gathered}
b^{11}=b_{p}^{1} \cdot \tau, \quad b^{12}=b_{p}^{1} \cdot \gamma_{2}, \quad b^{21}=b_{p}^{2} \cdot \tau, \quad b^{22}=b_{p}^{2} \cdot \gamma_{2}, \\
\alpha^{11}=a^{i j} \tau^{i} \tau^{j}, \quad \alpha^{12}=a^{i j} \tau^{i} \gamma_{2}^{j}, \quad \alpha^{22}=a^{i j} \gamma_{2}^{i} \gamma_{2}^{j}
\end{gathered}
$$

and note that $b^{22}>0,\left|\alpha^{11}\right|+\left|\alpha^{12}\right| \leq 2 \mu\left|\alpha^{22}\right|$. It follows that there is a bounded function $\psi$ such that

$$
\begin{aligned}
M^{*} v= & \frac{1}{b^{22}}\left[T\left(b^{11} b^{22}-b^{12} b^{21}\right)+\frac{2 \alpha^{12} b^{12} b^{21}}{\alpha^{22}}\right. \\
& \left.-\frac{b^{22} b^{12} \alpha^{11}}{\alpha^{22}}-b^{11} b^{21}\right] D_{i j} u \tau^{i} \tau^{j} \\
& +(1+|T|) \psi+\left(h_{t}+L_{0}\right) f^{\prime} M^{*} w
\end{aligned}
$$

From (2.1c), we have

$$
\left|b^{11} b^{22}-b^{12} b^{21}\right| \geq \varepsilon \text { on } \Sigma_{R}^{2}
$$

and hence we can choose $T$ with $|T| \leq C$ so that the coefficient of $D_{i j} u \tau^{i} \tau^{j}$ in $M^{*} v$ vanishes. With this choice of $T$, we are ready to 
choose $w$. From [17, Lemma 2] there are positive constants $\delta, C^{\prime}$, and $R_{0}$ and a function $w_{0}$ such that

$$
\begin{gathered}
F^{i j} D_{i j} w_{0} \leq-|x|^{\delta-2}, \quad|x|^{\delta} \leq w_{0} \leq C^{\prime}|x|^{\delta} \quad \text { in } \Omega_{R_{0}}, \\
M^{*} w \leq-|x|^{\delta-1} \text { on } \Sigma_{R_{0}}^{2} .
\end{gathered}
$$

If we write $\rho_{k}$ for the regularized distance to $\Sigma^{k}$, it is easy to verify that, for appropriate constants $A$ and $W$, the functions

$$
w_{k}=A \int_{0}^{\rho_{k}} \exp \left(-W \sigma^{\alpha}\right) d \sigma
$$

satisfy

$$
\begin{gathered}
F^{i j} D_{i j} w_{k} \leq-d_{k}^{\alpha-1}, \quad 0 \leq w_{k} \leq C^{\prime \prime}|x|^{\delta} \quad \text { in } \Omega, \\
M^{*} w_{k} \leq C^{\prime \prime}|x|^{\delta-1} \quad \text { on } \Sigma^{2} .
\end{gathered}
$$

Hence $w=\left(1+2 C^{\prime \prime}\right) w_{0}+w_{1}+w_{2}$ satisfies

$$
\begin{gathered}
F^{i j} D_{i j} w \leq-d^{\alpha-1}, \quad 0 \leq w \leq C|x|^{\delta} \text { in } \Omega_{R_{0}}, \\
M^{*} w \leq-|x|^{\delta-1} \quad \text { on } \Sigma_{R}^{2} \cap \Sigma_{R_{0}}^{2} .
\end{gathered}
$$

We now fix $R=\min \left\{R_{0}, \varepsilon /(2+2 K)\right\}, \eta \in(0,1)$ and, for $f_{0}, \beta$ positive constants to be chosen, we set

$$
f(w)=f_{0}\left(1+M R^{-\beta \delta}\right)(w+\eta)^{\beta} .
$$

If $f_{0}$ is chosen sufficiently large (in particular so that $f>1$ on $|x|=R$ ) and $L_{0}, c_{0}$ are as in Theorem 1.1, we see that

$$
\begin{gathered}
F^{i j} D_{i j} v-\frac{c_{0}}{f} D_{i} v D_{i} v<0 \text { in } \Omega^{*}=\Omega \cap\{f<1\}, \\
v \geq 0 \text { on } \sigma^{*}=\partial \Omega^{*} \backslash\left(\Sigma^{2} \cup\{0\}\right), \\
M^{*} v<0 \text { on } \Sigma^{*}=\partial \Omega^{*} \cap \Sigma^{2}
\end{gathered}
$$

If we set $L=F^{i j} D_{i j}-\left(c_{0} / f\right) D_{i} v D_{i}$ and note that

$$
\frac{c_{0}}{f}|D v| \leq \frac{c_{1}(\eta)}{|x|}
$$

we infer from [17, Lemma 2] that there are a function $\bar{w}$ and a positive constant $\theta$, both depending on $\eta$ such that

$$
\begin{gathered}
L \bar{w} \leq 0, \quad \bar{w} \geq|x|^{-\theta} \quad \text { in } \Omega^{*}, \\
M^{*} \bar{w} \leq 0 \quad \text { on } \Sigma^{*} .
\end{gathered}
$$


Now we fix $\eta_{1}>0$ and choose $r$ so that

$$
v+\eta_{1} \bar{w} \geq 0 \text { for }|x| \leq r .
$$

It then follows from the maximum principle that

$$
v+\eta_{1} \bar{w} \geq 0 \text { in } \Omega^{*} .
$$

By sending $\eta_{1}$ to zero, we see that $v \geq 0$ in $\Omega^{*}$. By sending $\eta$ to zero and modifying the argument appropriately, we see that

$$
\left|b^{k}(0, u(0), D u)\right| \leq C(1+M)|x|^{\delta \beta} .
$$

Then the implicit function theorem implies that

$$
|D u| \leq C(1+M)|x|^{\delta \beta}
$$

which proves the result.

Note that the preceding argument simplifies if we assume $D u$ to be continuous at 0 : we can take $\eta=0$ and $\bar{w}$ need not be introduced at all. Note also that the smoothness of $b^{i}$ is crucial to the proof of Theorem 2.1 but only the quantities $\varepsilon, \varepsilon_{0}$, and $\nu_{1}$ enter into the estimate. If we allow $C$ to depend on the $C^{1, \alpha}$ norm of $b^{i}$ in (2.3), then the bootstrap argument of [19, Theorem 2] shows that $\sigma$ depends only on $\nu_{1}, \mu, \varepsilon$, and $\theta_{0}$. Moreover it follows from the $C^{1, \alpha}$ estimate for $b^{1}$ that $\left|D^{2} u\right|=0\left(|x|^{\sigma-1}\right)$ and hence we can prove $(2.3)$ if only $b^{2}$ is oblique; if only $b^{1}$ is oblique, a similar result holds. Finally the limiting cases $\theta_{0}=0$ and $\theta_{0}=\pi$ are handled by a simple modification of the preceding arguments.

3. The two-dimensional capillary problem. We now consider a slight generalization of $(0.1)$. Let $\Omega$ be a bounded open subset of $\mathbf{R}^{2}$, suppose $\partial \Omega$ is the union of finitely many $C^{3}$ curves $\Sigma_{1}, \ldots, \Sigma_{N}$, and suppose that if $\Sigma_{i}$ and $\Sigma_{j}$ meet, they do so at a point $x_{i j}$ in an angle $\theta_{i j}$. We then study solutions of

$$
\operatorname{div}\left(\left(1+|D u|^{2}\right)^{-1 / 2} D u\right)+H(x, u)=0 \quad \text { in } \Omega,
$$

$$
\left(1+|D u|^{2}\right)^{-1 / 2} D u \cdot \gamma+\cos \phi(x)=0 \quad \text { on } \partial \Omega \backslash \bigcup\left\{x_{i j}\right\},
$$

assuming

$$
\phi \in C^{2}, \quad H \in C^{0,1},
$$

$$
|\phi|_{C^{2}\left(\Sigma_{\imath}\right)} \leq \Phi_{2}, \quad|H|_{0,1} \leq H_{0}
$$




$$
H_{z} \leq-\kappa, \quad \Phi_{0} \leq \phi \leq \pi-\Phi_{0}
$$

for some positive constants $\kappa, \Phi_{0}$. We also need to assume condition (2.2). To state this condition in the present context, we set

$$
\phi_{1}=\lim _{\substack{x \rightarrow x_{i j} \\ x \in \Sigma_{i}}} \phi(x), \quad \phi_{2}=\lim _{\substack{x \rightarrow x_{i j} \\ x \in \Sigma,}} \phi(x)
$$

and then (2.2) becomes

$$
I=\min _{i, j}\left\{\theta_{i j}-\left(\left|\phi_{1}-\frac{\pi}{2}\right|+\left|\phi_{2}-\frac{\pi}{2}\right|\right)\right\}>0 .
$$

A straightforward calculation shows that

$$
b^{11} b^{22}-b^{12} b^{21}=-\left(1+|p|^{2}\right)^{-1} \sin \theta_{i j},
$$

$$
T=\left\{\cos \theta_{i j}\left(1+\cos \phi_{2}^{2}\right)+\cos \phi_{1} \cos \phi_{2}\right\} / \sin \theta_{i j}\left(1+\left|p^{*}\right|^{2}\right) .
$$

From [4] and [13, Theorem 4.2] it follows that $u$ and $D u$ are bounded and hence Theorem 2.1 and its attendant remarks give an existence, uniqueness and regularity theorem.

THEOREM 3.1. Under conditions (3.2), (3.3), if $\Sigma_{i} \in C^{3}$, then (3.1) has a unique solution $u \in C^{1}\left(\bar{\Omega} \backslash \bigcup\left\{x_{i j}\right\}\right) \cap C^{0,1}(\bar{\Omega}) \cap C^{2}(\Omega)$. Moreover there is a positive constant $\delta$ determined only by the quantities $\theta_{i j}, \phi_{1}$ and $\phi_{2}$ such that $u \in C^{1, \delta}$ and

$$
|D u|_{\delta} \leq C\left(I, \Omega, H_{0}, \Phi_{2}, \Phi_{0}, \phi_{1}, \phi_{2}\right) .
$$

The continuity of $D u$ at each $x_{i j}$ was proved by Simon [25] under much weaker hypotheses. The new elements here are the simple proof and the explicit modulus of continuity. In [23, §4] Miersemann proves Theorem 3.1 for constant $H$ by introducing an auxiliary Dirichlet problem. Because we only need $H_{z}<0$ for a bound on $|u|$, our result includes his.

Actually all of our additional hypotheses are necessary only to prove a bound on $D u$. If we assume such a bound, a stronger result can be proved although deeper methods must be used. This result will be crucial to our higher-dimensional considerations, so we include it here.

THEOREM 3.2. Let $\partial \Omega$ be the union of finitely many $C^{1, \alpha}$ curves $\Sigma_{1}, \ldots, \Sigma_{N}$ and suppose that if $\Sigma_{i}$ and $\Sigma_{j}$ meet they do so at a point $x_{i j}$ 
in an angle $\theta_{i j} \in(0, \pi)$. Let $u \in C^{0}(\bar{\Omega}) \cap W^{1,2}(\Omega)$ be a weak solution of

$$
\begin{gathered}
\operatorname{div} A(x, u, D u)+B(x, u, D u)=0 \quad \text { in } \Omega, \\
A(x, u, D u) \cdot \gamma+\psi(x, u)=0 \quad \text { on } \partial \Omega
\end{gathered}
$$

suppose

$$
A\left(x_{i j}, u\left(x_{i j}\right), 0\right)=0, \quad \psi\left(x_{i j}, u\left(x_{i j}\right)\right)=0,
$$

and suppose there are positive constants $q>1, \mu, \mu_{0}, \mu_{1}, \mu_{2}$ and $a$ function $b \in L^{q}(\Omega)$ such that

$$
\begin{gathered}
|A(x, z, p)-A(y, w, p)| \leq \mu_{1}\left(|x-y|^{\alpha}+|z-w|^{\alpha}\right)(1+|p|) \\
|\psi(x, z)-\psi(y, w)| \leq \mu_{2}\left(|x-y|^{\alpha}+|z-w|^{\alpha}\right) \\
|\xi|^{2} \leq \frac{\partial A^{i}}{\partial p_{j}}(x, z, p) \xi_{i} \xi_{j} \leq \mu|\xi|^{2} \\
|B(x, z, p)| \leq \mu_{0}|p|^{2}+b(x) \\
\int_{B(r) \cap \Omega} b^{q} \leq \mu_{0}^{q} r^{q \alpha-q+2}
\end{gathered}
$$

for all balls $B(r)$ centered in $\Omega$. Then $u \in C^{1}(\bar{\Omega})$ and there are positive constants $C$ and $\beta$ determined by $|u|_{0}, \Omega, \mu, \mu_{0}, \mu_{1}, \mu_{2}, q$ such that

$$
|D u|_{\beta} \leq C \text {. }
$$

Proof. To prove the estimate, we use a modification of the Campanato approach to regularity [1], [2] as adapted by Giaquinta and Giusti [8] and the author [20].

We begin with a simpler boundary value problem. Write $r, \theta$ for polar coordinates, fix $\theta_{0} \in(\theta, \pi)$, and for $R>0$, set

$$
\begin{aligned}
& \Omega_{R}=\left\{r<R, 0<\theta<\theta_{0}\right\}, \\
& \Sigma_{R}^{1}=\left\{0<r<R, \theta=\theta_{0}\right\}, \quad \Sigma_{R}^{2}=\{0<r<R, \theta=0\}, \\
& \Sigma_{R}=\Sigma_{R}^{1} \cup \Sigma_{R}^{2} \cup\{0\}, \quad \sigma_{R}=\left\{r=R, 0 \leq \theta \leq \theta_{0}\right\} .
\end{aligned}
$$

Suppose $H \in C^{2}\left(\mathbf{R} \backslash\{0\} \times \mathbf{R}^{2}\right) \cap C^{0}\left(\mathbf{R} \times \mathbf{R}^{2}\right)$ is a vector valued function such that

$$
\begin{aligned}
& H(0,0)=0, \quad \frac{\partial H^{i}}{\partial p_{j}}(t, p) \xi_{i} \xi_{j} \geq|\xi|^{2}, \\
& |D H(t, p)| \leq \mu, \quad\left|D^{2} H(t, p)\right| \leq \frac{\mu}{|t|}
\end{aligned}
$$


for all $t \in \mathbf{R} \backslash\{0\}, p \in \mathbf{R}^{2}$. Fix $R \in(0,1)$, let $u \in W^{1,2}\left(\Omega_{2 R}\right)$, and set $M=(1 / R)\|D u\|_{L^{2}\left(\Omega_{2 R}\right)}$. Finally, suppose $v$ is a $C^{1}\left(\Omega_{2 R} \cup \Sigma_{2 R}\right) \cap$ $W^{1,2}\left(\Omega_{2 R}\right)$ solution of $\operatorname{div} H(M R, D v)=0 \quad$ in $\Omega_{2 R}$,

$$
H(M R, D v) \cdot \gamma=0 \quad \text { on } \Sigma_{2 R}, \quad v=u \text { on } \sigma_{2 R} .
$$

It follows that

$$
\int_{\Omega_{2 R}} H(M R, D v) \cdot D \phi=0
$$

for any $\phi \in W^{1,2}\left(\Omega_{2 R}\right)$ vanishing on $\sigma_{2 R}$. By choosing $\phi=u-v$, we find that

$$
\int_{\Omega_{2 R}}|D v|^{2} \leq c(\mu) M^{2} R^{2}
$$

Next we keep track of the way in which $R$ and $M$ enter into the gradient estimates of Ladyzhenskaya and Ural'tseva [14, Chapter 10] (cf. [9, Theorem 8.16] and [20, Lemma 4.1]) to see that

$$
\sup _{\sigma_{R}}|D v| \leq c_{1} M .
$$

We now set

$$
\bar{v}=\gamma_{1} \cdot H(f(w), D v)+L_{0} f(w), \quad a_{1}=\gamma_{1} \cdot H_{t}(f(w), D v)
$$

in $\Omega_{R}$, with $w$ as in Theorem 2.1 and $L_{0}, f$ to be chosen. From the proof of that theorem, we can choose $T$ so that, if $M^{*}=T D_{1}+D_{2}$, then

$$
M^{*} \bar{v}=f^{\prime}(w)\left(L_{0}+a_{1}\right) M^{*} w .
$$

Because $|T|+\left|a_{1}\right| \leq C\left(\mu, \theta_{0}\right)$ it follows that we can determine $w$ and constants $\delta, c^{\prime}$ in terms of $\mu, \theta_{0}$ so that

$$
\begin{gathered}
M^{*} w \leq-r^{\delta-1} \text { on } \Sigma_{R}^{2}, \\
a^{i j} D_{i j} w \leq-r^{\delta-2}, \quad 0 \leq w \leq c^{\prime} r^{\delta} \text { in } \Omega_{R}
\end{gathered}
$$

for

$$
a^{i j}=\frac{\partial H^{i}}{\partial p_{j}}(M R, D v)
$$

Standard regularity implies that $v \in W_{\text {loc }}^{3,2}$ and hence that $\bar{v} \in W_{\text {loc }}^{2,2}$. Thus, if $f(w) \geq M R$, there are nonnegative constants $c_{2}$ and $c_{3}$ such 
that

$$
\begin{aligned}
Q \bar{v}= & a^{i j} D_{i j} v-\frac{c_{2}}{f}|D \bar{v}|^{2} \\
\leq & a^{i j} D_{i} w D_{j} w\left[\left(L_{0}+a_{1}\right) f^{\prime \prime}+c_{3}\left(1+L_{0}^{2}\right) \frac{\left(f^{\prime}\right)^{2}}{f}\right] \\
& +a^{i j} D_{i j} w\left[\left(L_{0}+a_{1}\right) f^{\prime}\right] .
\end{aligned}
$$

We now choose $L_{0}=\left(2+c_{1}\right) \mu+1$ to find that

$$
M^{*} \bar{v}<0 \quad \text { on } \Sigma_{R}^{2}, \quad \bar{v} \geq 0 \quad \text { on } \partial M_{R} \backslash \Sigma_{R}^{2} .
$$

Finally we choose $\varepsilon$ sufficiently small and

$$
f(w)=M\left(R^{1 / \varepsilon}+w R^{-\delta}\right)^{\varepsilon}
$$

to obtain

$$
Q \bar{v}<0 \quad \text { in } \Omega_{R} .
$$

The maximum principle implies that $\bar{v} \geq 0$ in $\Omega_{R}$. If we replace $L_{0}$ by $-L_{0}$ and then $\gamma_{1}$ by $\gamma_{2}$ in the definition of $\bar{v}$, similar arguments show that

$$
|D v| \leq C M\left[R+\left(\frac{r}{R}\right)^{\delta \varepsilon}\right] \quad \text { in } \Omega_{R}
$$

Note that this estimate, along with standard results (see [20, Lemma 4.1]), implies that (3.11) has a $C^{1}\left(\Omega_{2 R} \cup \Sigma_{2 R}\right) \cap W^{1,2}\left(\Omega_{2 R}\right)$ weak solution, which is the unique $W^{1,2}\left(\Omega_{2 R}\right)$ weak solution.

We now use (3.12) to prove an estimate for $D u$ by using a version of the perturbation argument of Giaquinta and Giusti [8]. By virtue of the estimates in [20], we only need to examine $D u$ near one of the $x_{i j}$ 's. By translation and a $C^{1, \alpha}$ transformation, we may assume that

$$
\operatorname{div} A(x, u, D u)+B(x, u, D u)=0 \quad \text { in } \Omega_{2},
$$

$$
A(x, u, D u) \cdot \gamma+\psi(x, u)=0 \text { on } \Sigma_{2}
$$

Let $H$ be the mollification of $A(0, u(0), \cdot)$ described in Theorem 1.1. Now fix $R \in(0,1)$ and let $v$ be the solution of (3.11). From (3.12) we infer that

$$
\int_{\Omega_{\rho}}|D v|^{2} \leq C\left[\rho^{2}+\left(\frac{\rho}{2 R}\right)^{2 \delta \varepsilon+2}\right] \int_{\Omega_{2 R}}|D u|^{2}
$$


for any $\rho \in(0,2 R)$. By using $u-v$ as test function in the weak forms of (3.11) and (3.13) we see that

$$
\begin{aligned}
\int_{\Omega_{2 R}}|D u-D v|^{2} \leq & \int_{\Omega_{2 R}}\{H(0, D u)-H(0, D v)\} \cdot\{D u-D v\} \\
= & \int_{\Omega_{2 R}}\{H(0, D u)-A(x, u, D u)\} \cdot\{D u-D v\} \\
& +\int_{\Omega_{2 R}} B(x, u, D u)(u-v)+\int_{\Sigma_{2 R}} \psi(x, u)(u-v) \\
& +\int_{\Omega_{2 R}}\{H(M R, D v)-H(0, D v)\} \cdot\{D u-D v\} .
\end{aligned}
$$

Let us write $I_{1}, I_{2}, I_{3}, I_{4}$ for the integrals on the right hand side of this equation. From the Hölder estimate for $u([14$, p. 467]), it follows that there is a positive $\eta$ such that

$$
I_{1} \leq C R^{\alpha \eta} \int_{\Omega_{2 R}}(1+|D u|)|D u-D v| .
$$

A simple modification of [9, Theorem 10.9] shows that

$$
\sup _{\Omega_{2 R}}\left(v-\sup _{\Omega_{2 R}} u\right), \quad \sup _{\Omega_{2 R}}\left(\inf _{\Omega_{2 R}} u-v\right) \leq C M R^{2}
$$

and hence because $\int_{\Omega_{2}}|D u|^{2} \leq C$,

$$
\sup |u-v| \leq C R^{\eta}
$$

Therefore

$$
\begin{aligned}
I_{2} & \leq C R^{\eta} \int|D u|^{2}+\mu_{0} R^{\alpha-1+2 / q}\left(\int|u-v|^{q /(q-1)}\right)^{1-(1 / q)} \\
& \leq C R^{\eta} \int|D u|^{2}+C R^{\alpha+1}\left(\int|D u-D v|^{2}\right)^{1 / 2}
\end{aligned}
$$

by Sobolev's inequality. Also

$$
\begin{aligned}
I_{3} & \leq C R^{\alpha \eta} \int_{\Sigma_{2 R}}|u-v| \\
& \leq C R^{\alpha \eta} \int_{\Omega_{2 R}}|D u-D v| \leq C R^{\alpha \eta+1}\left(\int_{\Omega_{2 R}}|D u-D v|^{2}\right)^{1 / 2}
\end{aligned}
$$

and

$$
I_{4} \leq C\left(\int_{\Omega_{2 R}}|D u|^{2}\right)^{1 / 2} R\left(\int_{\Omega_{2 R}}|D u-D v|^{2}\right)^{1 / 2}
$$


Hence

$$
\int_{\Omega_{2 R}}|D u-D v|^{2} \leq C R^{2 \alpha \eta+2}+C R^{\eta} \int_{\Omega_{2 R}}|D u|^{2} .
$$

In conjunction with (3.14), this inequality implies that

$$
\int_{\Omega_{\rho}}|D u|^{2} \leq C\left[R^{\eta}+\left(\frac{\rho}{R}\right)^{2 \delta \varepsilon+2}\right] \int_{\Omega_{2 R}}|D u|^{2}+C R^{2 \alpha \eta+2} .
$$

It follows that

$$
\int_{\Omega_{R}}|D u|^{2} \leq C_{0}(\sigma) R^{2 \sigma+2}
$$

for any $\sigma<\min \{\delta \varepsilon, \alpha \eta\}$ and $R \in(0,2)$.

For $x_{0} \in \Omega_{1}$, set $B_{\rho}^{+}\left(x_{0}\right)=\left\{x \in \Omega:\left|x-x_{0}\right|<\rho\right\}$ and

$$
\{u\}_{\rho}=\frac{1}{\left|B_{\rho}^{+}\left(x_{0}\right)\right|} \int_{B_{\rho}^{+}\left(x_{0}\right)} u .
$$

Now choose $\tau=\tau\left(\theta_{0}\right) \in(0,1)$ so that if $x_{0} \in \Sigma_{1}^{1}$, then

$$
B_{\tau\left|x_{0}\right|}^{+}\left(x_{0}\right) \cap \Sigma_{1}^{2}=\varnothing \text {. }
$$

It then follows from the proof of [20, Theorem 5.1] that

$$
\int_{B_{\rho}^{+}\left(x_{0}\right)}\left|D u-\{D u\}_{\rho}\right|^{2} \leq C\left(\frac{\rho}{\left|x_{0}\right|}\right)^{2 \lambda+2} \int_{B_{\tau\left|x_{0}\right|}\left(x_{0}\right)}\left|D u-\{D u\}_{\rho}\right|^{2}+C \rho^{2 \alpha \eta+2}
$$

for some $\lambda \in(0, \alpha)$ and all $\rho \in\left(0, \tau\left(x_{0}\right)\right), x_{0} \in \Sigma_{1} \backslash\{0\}$. Combining this inequality with $(3.16)$ yields

$$
\int_{B_{\rho}^{+}\left(x_{0}\right)}\left|D u-\{D u\}_{\rho}\right|^{2} \leq C \rho^{2 \sigma+2}
$$

if also $\sigma \leq \lambda$. A similar argument shows that this inequality is valid for any $x_{0} \in \Omega_{3 / 2}$ and $\rho \in(0,2)$. Then Campanato's result shows that

$$
[D u]_{\sigma ; \Omega_{3 / 2}} \leq C \text {. }
$$

In particular $|D u|$ is bounded so we can repeat the preceding argument with $\eta=1$ to conclude

$$
[D u]_{\sigma ; \Omega_{1}} \leq C
$$

for $\sigma<\min \{\delta \varepsilon, \lambda\}$. In conjunction with the Hölder estimate for $D u$ away from the $x_{i j}$ 's, this inequality proves the result.

We note that a slight variant of the preceding proof (cf. [8] or [20, Theorem 1.2]) shows that $\beta=\min \{\delta, \alpha\}$ when $\partial A / \partial p$ depends continuously on $p$. In this case, $C$ depends also on the modulus of continuity. 
Finally, by using the $C^{1}$ regularity from [25], we infer from Theorem 3.2 that bounded weak solutions of (3.1) are $C^{1, \delta}$ with $\delta$ determined by $\theta_{i j}, \phi_{1}, \phi_{2}$, and $\alpha$ if the $\Sigma_{i}$ 's are $C^{1, \alpha}$, if (3.3) holds, and if (3.2) is replaced by

$$
\phi \in C^{\alpha}, \quad H \in L^{\infty}, \quad \Phi_{0} \leq \phi \leq \pi-\Phi_{0} .
$$

4. The higher-dimensional capillary problem. Like the geometric measure theoretical approaches of Simon [25] and Tam [26], our method relies on special features of the two-dimensional situation although the specific features used vary among the three approaches. Our goal in this section is to show how to handle higher-dimensional situations.

The natural analog of a corner turns out to be an edge in the present context. We begin by introducing a convenient shorthand notation. We say that $\Omega \in E\left(\alpha, \theta_{1}\right)$ for $\alpha \in(0,1), \theta_{1} \in(0, \pi / 2)$ if $\partial \Omega$ is the union of finitely many $C^{1, \alpha}$ surfaces $\Sigma_{1}, \ldots, \Sigma_{N}$ such that if $\Sigma_{i}$ and $\Sigma_{j}$ meet, they do so at an $(n-2)$-dimensional surface $\Sigma_{i j}$ and at each $x_{i j} \in \Sigma_{i j}$, the angle between $\Sigma_{i}$ and $\Sigma_{j}$ lies in the range $\left(\theta_{1}, \pi-\theta_{1}\right)$.

THEOREM 4.1. Let $\Omega \in E\left(\alpha, \theta_{1}\right)$ be a bounded open subset of $\mathbf{R}^{n}$, $n \geq 3$. Let $u \in C^{0}(\bar{\Omega}) \cap W^{1,2}(\Omega)$ be a weak solution of (3.7). Suppose that

$$
\psi\left(x_{i j}, u\left(x_{i j}\right)\right)=0 \text { for all } x_{i j} \in \Sigma_{i j}
$$

and that for any vector $\tau_{i j}$ tangent to $\Sigma_{i j}$ at $x_{i j}$, there is a vector $\nu_{i j}$ normal to $\Sigma_{i j}$ there such that

$$
A\left(x_{i j}, u\left(x_{i j}\right), \tau_{i j}+\nu_{i j}\right) \cdot \gamma_{k}\left(x_{i j}\right)=0 \text { for } k=i, j .
$$

Suppose that there are positive constants $q>n / 2, \mu, \mu_{0}, \mu_{1}, \mu_{2}$ and $a$ function $b \in L^{q}(\Omega)$ such that $(3.9 \mathrm{a}-\mathrm{d})$ and

$$
\int_{B(r) \cap \Omega} b^{q} \leq \mu_{0}^{q} r^{q \alpha-q+n}
$$

hold for all balls $B(r)$ centered in $\Omega$. Then $u \in C^{1}(\bar{\Omega})$ and there are positive constants $C$ and $\beta$ determined by $|u|_{0}, \Omega, \mu, \mu_{0}, \mu_{1}, \mu_{2}, q$ for which (3.10) holds.

Proof. We look locally near $\Sigma_{i j}$. For $R>0$ and $\theta_{0} \in(0, \pi)$ set

$$
\begin{aligned}
W_{R} & =\left\{x=\left(x^{\prime}, x^{\prime \prime}\right) \in \mathbf{R}^{2} \times \mathbf{R}^{n-2}:\left|x^{\prime \prime}\right|<R, x^{\prime} \in \Omega_{R}\right\}, \\
S_{R} & =\left\{\left(x^{\prime}, x^{\prime \prime}\right):\left|x^{\prime \prime}\right|<R, x^{\prime} \in \Sigma_{R}\right\}, \\
s_{R} & =\left\{\left(x^{\prime}, x^{\prime \prime}\right):\left|x^{\prime \prime}\right|<R, x^{\prime} \in \sigma_{R}\right\}, \\
S_{R}^{\prime} & =\left\{\left(x^{\prime}, x^{\prime \prime}\right):\left|x^{\prime \prime}\right|=R, x^{\prime} \in \bar{\Omega}_{R}\right\},
\end{aligned}
$$


where $\Sigma_{R}, \sigma_{R}, \Omega_{R}$ were defined in the proof of Theorem 3.2. We may assume as before that $u$ is a weak solution of

$$
\begin{gathered}
\operatorname{div} A(x, u, D u)+B(x, u, D u)=0 \quad \text { in } W_{2}, \\
A(x, u, D u) \cdot \gamma+\psi(x, u)=0 \quad \text { on } S_{2} .
\end{gathered}
$$

Our first step is to show that $D^{\prime \prime} u$ is Hölder continuous. Fix $R \in(0,2)$ and let $v$ solve

$$
\operatorname{div} A(0, u(0), D v)=0 \quad \text { in } W_{R}
$$

$$
A(0, u(0), D v) \cdot \gamma=0 \quad \text { on } S_{R}, \quad v=u \quad \text { on } s_{R} \cup S_{R}^{\prime} .
$$

A standard difference quotient argument implies that $D_{k} v(k=3, \ldots$, $n$ ) is a weak solution of the problem

$$
D_{i}\left(a^{i j} D_{j} w\right)=0 \quad \text { in } W_{R}, \quad a^{i j} D_{j} w \gamma^{i}=0 \quad \text { on } S_{R}
$$

for $a^{i j}=\left(\partial A^{i} / \partial p_{j}\right)(0, u(0), D v)$ and hence (cf. [9, Theorem 8.22])

$$
\underset{W_{\rho}}{\operatorname{osc}} D_{k} v \leq C\left(\frac{\rho}{R}\right)^{\sigma} \underset{W_{R}}{\operatorname{osc}} D_{k} v
$$

for some $\sigma\left(\mu, \theta_{1}\right)>0$. As in the proof of [20, (4.9)] it follows that

$$
\int_{W_{\rho}}\left|D_{k} v-\left\{D_{k} v\right\}_{\rho}\right|^{2} \leq C\left(\frac{\rho}{R}\right)^{2 \sigma+n} \int_{W_{R}}\left|D_{k} v-\left\{D_{k} v\right\}_{R}\right|^{2} .
$$

From this estimate, it follows that $D^{\prime \prime} u \in C^{\delta}$ if $\delta<\min \{\alpha, \sigma\}$, with $\left|D u^{\prime \prime}\right|_{\delta} \leq C$. Thus we may assume that

$$
D^{\prime \prime} u=0 \text { on }\left\{\left|x^{\prime}\right|=0\right\}, \quad \tau_{i j}=0 \Rightarrow \nu_{i j}=0,
$$

$A^{1}$ and $A^{2}$ are independent of $D^{\prime \prime} u$,

and $(3.9 \mathrm{a}-\mathrm{d}),(4.2)$ are valid with $\delta$ replacing $\alpha$.

Under these assumptions, we return to (4.3). Let us set

$$
\begin{gathered}
a^{i}\left(p^{1}, p^{2}\right)=A^{i}\left(0, u(0), p^{1}, p^{2}\right), \quad i=1,2, \\
f=\sum_{i>2} \sum_{j=1}^{n} \frac{\partial A^{i}}{\partial p_{j}}(0, u(0), D v) D_{i j} v .
\end{gathered}
$$

By virtue of (4.4), $f \in L^{2}\left(W_{R / 2}\right)$ and

$$
\int_{W_{R / 2}}|f|^{2} \leq C R^{-2} \int_{W_{R}}\left|D^{\prime \prime} v\right|^{2}
$$


Hence for almost all $x^{\prime \prime} \in\left\{\left|x^{\prime \prime}\right|<R / 2\right\}, f\left(\cdot, x^{\prime \prime}\right) \in L^{2}\left(\Omega_{R / 2}\right)$ by Fubini's theorem and $v\left(\cdot, x^{\prime \prime}\right)$ is a weak solution of

$$
\begin{gathered}
\operatorname{div} a\left(D^{\prime} v\left(\cdot, x^{\prime \prime}\right)\right)+f\left(\cdot, x^{\prime \prime}\right)=0 \quad \text { in } \Omega_{R / 2}, \\
a\left(D^{\prime} v\left(0, x^{\prime \prime}\right)\right) \cdot \gamma=0 \quad \text { on } \Sigma_{R / 2} .
\end{gathered}
$$

In addition, if $M=R^{-n / 2}\|D u\|_{L^{2}\left(W_{R}\right)}$, we have as before that $|D v| \leq$ $C M$ on $S_{R / 2}$. An easy variant of the proof of Theorem 3.2 shows that there is a positive constant $\eta$ such that

$$
\int_{\Omega_{\rho}}\left|D^{\prime} v\left(\cdot, x^{\prime \prime}\right)\right|^{2} \leq C\left[\left(\frac{\rho}{R}\right)^{2 \eta+2}+R^{2}\right] M^{2} R^{2}+C R^{2} \int_{\Omega_{R / 2}}\left|f\left(\cdot, x^{\prime \prime}\right)\right|^{2}
$$

By integrating this inequality with respect to $x^{\prime \prime}$, we see that

$$
\begin{aligned}
\int_{W_{\rho}}\left|D^{\prime} v\right|^{2} & \leq C\left[\left(\frac{\rho}{R}\right)^{2 \eta+2}+R^{2}\right] M^{2} R^{2} \rho^{n-2}+C \int_{W_{R}}\left|D^{\prime \prime} v\right|^{2} \\
& \leq C\left[\left(\frac{\rho}{R}\right)^{2 \eta+n}+R^{2}\right] \int_{W_{R}}|D u|^{2}+C \int_{W_{R}}\left|D^{\prime \prime} v\right|^{2} .
\end{aligned}
$$

This estimate implies that

$$
\int_{W_{R}}\left|D^{\prime} u\right|^{2} \leq C R^{2 \varepsilon+n}
$$

for some positive $\varepsilon$, and the Hölder estimate for $D^{\prime} u$ follows as before.

Theorem 4.1 applies to the capillary problem provided a gradient bound is known. Again [4] and [13, Theorem 4.2] give the required estimates. To state the condition on the edges, we define

$$
\phi_{k}\left(x_{i j}\right)=\lim _{\substack{x \rightarrow x_{i j} \\ x \in \Sigma_{k}}} \phi(x), \quad k=i, j,
$$

and write $\theta_{i j}\left(x_{i j}\right)$ for the angle between $\Sigma_{i}$ and $\Sigma_{j}$ at $x_{i j}$.

THEOREM 4.2. Let $\Omega \in E\left(\alpha, \theta_{1}\right)$ and suppose the $\Sigma_{i}$ 's are $C^{3}$. If conditions (3.2), (3.3) are satisfied and if

$$
I=\inf _{x_{i j} \in \Sigma_{i j}}\left\{\theta_{i j}\left(x_{i j}\right)-\left|\phi_{i}\left(x_{i j}\right)-\frac{\pi}{2}\right|-\left|\phi_{j}\left(x_{i j}\right)-\frac{\pi}{2}\right|\right\}>0,
$$

there is a unique weak solution $u \in C^{1}\left(\bar{\Omega} \backslash \cup \Sigma_{i j}\right) \cap C^{0,1}(\bar{\Omega}) \cap C^{2}(\Omega)$ of (3.1). Moreover there are positive constants $\delta$ and $C$ determined by $|D u|_{0}, \theta_{1}, I, \Omega, H_{0}, \Phi_{0}, \Phi_{2}$ such that $u \in C^{1, \delta}$ and $|D u|_{\delta} \leq C$. 


\section{REFERENCES}

[1] S. Campanato, Proprietà di Hölderianita di alcune classi di funzioni, Ann. Scuola Norm. Sup. Pisa III, 17 (1963), 175-188.

[2] Equazioni ellitiche de $I I^{0}$ ordine e spazi $\mathscr{L}^{(2, \lambda)}$, Ann. Mat. Pura Appl. IV, 69 (1965), 321-381.

[3] P. Concus and R. Finn, On the behavior of a capillary surface in a wedge, Proc. Nat. Acad. Sci., 63 (1969), 292-299.

[4] , On a capillary free surface in a gravitational field, Acta Math., 132 (1974), 207-224.

[5] M. Emmer, Esistenzá, unicitá e regolaritá nelle superifici di equilibrio nei capillari, Ann. Univ. Ferrara Sez VII, 18 (1973), 79-94.

[6] R. Finn and C. Gerhardt, The internal sphere condition and the capillary problem, Ann. Mat. Pura Appl. IV, 112 (1977), 13-31.

[7] C. Gerhardt, Global regularity of the solutions to the capillarity problem, Ann. Scuola Norm. Sup. Pisa IV, 3 (1976), 157-176.

[8] M. Giaquinta and E. Giusti, Differentiability of minima of nondifferentiable functions, Inv. Math., 72 (1983), 285-298.

[9] D. Gilbarg and N. S. Trudinger, Elliptic Partial Differential Equations of Second Order, 2nd ed., Springer-Verlag, Berlin, 1983.

[10] E. Giusti, Boundary behavior of non-parametric minimal surfaces, Indiana Univ. Math. J., 22 (1972), 435-444.

[11] D. Kinderlehrer and G. Stampacchia, An Introduction to Variational Inequalities and Their Applications, Academic Press, New York, 1980.

[12] N. J. Korevaar, On the behavior of a capillary surface at a reentrant corner, Pacific J. Math., 88 (1980), 379-385.

[13] , Maximum principle gradient estimates for the capillary problem, Comm. Partial Differential Equations, 13 (1988), 1-31.

[14] O. A. Ladyzhenskaya and N. N. Ural'tseva, Linear and Quasilinear Elliptic Equations, Academic Press, New York, 1968.

[15] G. M. Lieberman, Solvability of quasilinear elliptic equations with nonlinear boundary conditions, Trans. Amer. Math. Soc., 273 (1982), 753-765.

[16] , Regularized distance and its applications, Pacific J. Math., 117 (1985), 329-352.

[17] - Mixed boundary value problems for elliptic and parabolic equations of second order, J. Math. Anal. Appl., 113 (1986), 422-440.

[18] - The Dirichlet problem for quasilinear elliptic equations with continuously differentiable boundary data, Comm. Partial Differential Equations, 11 (1986), 167-229.

[19] _ Two-dimensional nonlinear boundary value problems for nonlinear elliptic equations, Trans. Amer. Math. Soc., 300 (1987), 287-295.

[20] Houlder continuity of the gradient of solutions of uniformly parabolic equations with conormal boundary conditions, Ann. Mat. Pura Appl., to appear.

[21] _ Oblique derivative problems in Lipschitz domains II. Discontinuous boundary values, to appear.

[22] J. H. Michael, Barriers for uniformly elliptic equations and the exterior cone condition, J. Math. Anal. Appl., 79 (1981), 203-217.

[23] E. Miersemann, On capillary free surfaces without gravity, Z. Anal. Anwendungen, 4 (1985), 429-436. 
[24] K. Miller, Extremal barriers on cones with Phragmèn-Lindelöf theorems and other applications, Ann. Mat. Pura Appl. IV, 90 (1971), 297-329.

[25] L. M. Simon, Regularity of capillary surfaces over domains with corners, Pacific J. Math., 88 (1980), 363-377.

[26] L. F. Tam, Regularity of capillary surfaces over domains with corners: borderline case, Pacific J. Math., 125 (1986), 469-482.

[27] N. N. Ural'ceva, Solvability of the capillary problem I, II, Vestnik Leningrad. Univ. no. 19 (1973), 54-64, no. 1 (1975), 143-149 [Russian], Englisn translation in Vestnik Leningrad Univ. Math., 6 (1979), 363-375, 8 (1980), 151-158.

Received March 27, 1987.

IOWA STAte UNIVERsity

AMES, IA 50011 



\section{PACIFIC JOURNAL OF MATHEMATICS}

\section{EDITORS}

V. S. VARADARAJAN

(Managing Editor)

University of California

Los Angeles, CA 90024

Herbert Clemens

University of Utah

Salt Lake City, UT 84112

R. FINN

Stanford University

Stanford, CA 94305
HERMANN FLASCHKA

University of Arizona

Tucson, AZ 85721

RAMESh A. GANGOLLI

University of Washington Seattle, WA 98195

VAUGHAN F. R. JONES

University of California

Berkeley, CA 94720
ROBION KIRBY

University of California

Berkeley, CA 94720

C. C. MOORE

University of California

Berkeley, CA 94720

HAROLD STARK

University of California, San Diego

La Jolla, CA 92093

\section{ASSOCIATE EDITORS}
R. AREnS
E. F. BECKENBACH
B. H. NEUMANN
F. WOLF
K. YOSHIDA (1906-1982)

\section{SUPPORTING INSTITUTIONS}

UNIVERSITY OF ARIZONA

UNIVERSITY OF OREGON

UNIVERSITY OF BRITISH COLUMBIA UNIVERSITY OF SOUTHERN CALIFORNIA

CALIFORNIA INSTITUTE OF TECHNOLOGY

UNIVERSITY OF CALIFORNIA

STANFORD UNIVERSITY

MONTANA STATE UNIVERSITY

UNIVERSITY OF HAWAII

UNIVERSITY OF NEVADA, RENO

UNIVERSITY OF TOKYO

NEW MEXICO STATE UNIVERSITY

UNIVERSITY OF UTAH

OREGON STATE UNIVERSITY

WASHINGTON STATE UNIVERSITY

UNIVERSITY OF WASHINGTON 


\section{Pacific Journal of Mathematics}

\section{Vol. 133, No. $1 \quad$ March, 1988}

John Anderson, Finitely generated algebras and algebras of solutions to partial differential equations $\ldots \ldots \ldots \ldots \ldots \ldots \ldots \ldots \ldots \ldots \ldots \ldots \ldots$

Junichi Aramaki, On an extension of the Ikehara Tauberian theorem . . . . . 13

Giacomo Monti Bragadin, Abstract Riemannian stratifications .......... 31

Lawrence James Brenton and Richard Hill, On the Diophantine equation

$1=\sum 1 / n_{i}+1 / \prod n_{i}$ and a class of homologically trivial complex

surface singularities .................................41

C. Bruce Hughes, Controlled homotopy topological structures $\ldots . \ldots \ldots \ldots 69$

Peter Wilcox Jones and Takafumi Murai, Positive analytic capacity but zero Buffon needle probability . ...........................999

Gary M. Lieberman, Hölder continuity of the gradient at a corner for the

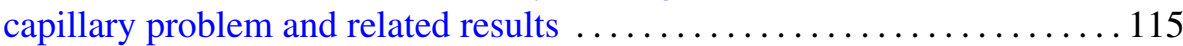

Feng Luo, Representing homology classes of $C \mathbf{P}^{2} \overline{C \mathbf{P}^{2}}$

Claudio Nebbia, Groups of isometries of a tree and the Kunze-Stein

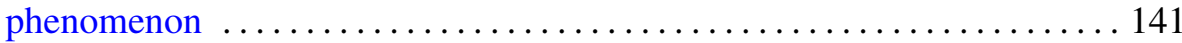

Stefan Richter, Unitary equivalence of invariant subspaces of Bergman and

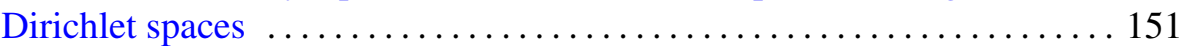

Paul Frederick Ringseth, The Selberg trace formula for groups without Eisenstein series ..................................... 157

Abderrazzak Sersouri, The Mazur property for compact sets $\ldots \ldots \ldots \ldots 185$ Alladi Sitaram, On an analogue of the Wiener Tauberian theorem for symmetric spaces of the noncompact type 